\title{
Study of Moineau-based pumps for the volumetric extrusion of pellets
}

\author{
Enrique Canessa, Marco Baruzzo and Carlo Fonda \\ Scientific Fabrication Laboratory (SciFabLab) \\ ICTP - The Abdus Salam International Centre for Theoretical Physics, Trieste, Italy \\ e-mail: scifablab@ictp.it
}

\begin{abstract}
We report on our on-going research to create a volumetric extruder for pellets or granules of recycled plastic to be used in a RepRap FDM 3D printer for rapid prototyping, by combining a rotor made of a Moineau progressing cavity pump coupled with an Auger screw, both contained inside a heated stator. We also introduce an alternative multi-layer Moineau-based pump -easier to build, implement and clean- for pumping a controlled quantity of viscous material in vertical direction. The latter, our screw pump model 131313, consists of a steer Auger portion which increases the pressure inside a layered double-helix stator-like container as a layered helical rotor-like is turned.
\end{abstract}

Keywords: 3D printing, RepRap, fluid dynamics, Moineau

\section{Introduction}

The geometry of a progressing cavity pump, or Moineau pump, is beautiful and simple in theory [1]. It consists of an inner (harder) helical rotor and an outer rubber (softer) stator which form a set of small- and fixed-size cavities in between. The rotor displaces viscous material throughout the system by means of the rotation of the cavities. The fluid can flow back or forward and the volumetric flow rate $Q$ is proportional to the rotation rate $N$ in both directions.

Progressing cavity pumps have many application areas including food, oil, etc. pumping (see, e.g., [2]). However, these type of pumps can present big challenges when applied in practice to small dimensions (only few centimetres in length) and at high temperatures (say, higher than 180 Celsius degrees). One novel application for using Moineau-based pumps with such extreme characteristics could found fertile grounds in the rapid production of 3D objects printed directly from thermoplastic pellets or granules of recycled plastic instead than from filament.

We discuss here on the use of a Moineau pump combined with an Auger pump (Archimede's screw) as first stage, both $3 \mathrm{D}$ printed out of aluminum, for the volumetric extrusion of pellets. In the following, we also describe in detail our first prototype for a new class of layered screw-like pump. Motivated by the aforementioned implementation constraints, we have sectioned the simplest (2:1) Moineau's progressive cavity pump in several, equally spaced, horizontal layers in order to re-design an alternative multi-layer pump system simplified to just a few layers that can be easier to build, assemble and clean, and get in practice, a reasonable continuous pumping of viscous material.

\section{Implementation of a thermoplastic extruder using a Moineau pump}

The first idea to implement a plastic extruder using a Moineau pump was inspired by an article appeared in Issue 3 of "RepRap Magazine" at page 26, entitled "The road to better paste extrusion" [3]. The author, Dries Verbruggen, explained the importance of volumetric extrusion and listed, among other solutions, two possible "positive displacement extruders": peristaltic pump and gear pump, comparing them to the non-volumetric Auger pump, but also mentioning briefly the Moineau pump at the very end of the article. After some more literature research, we decided to investigate further on this technology, since a $(n+1: n)$ Moineau is the only pump that can provide a volumetric extrusion and $\neg$ at the same time $\neg$ heating and melting the plastic (the cited article [3] was focused on the extrusion of paste, and it did not consider the heating part).

With the help of the very detailed mathematical analysis of Moineau pumps found in the article "The geometry of the Moineau pump" by Jens Gravensen, and of some additional on-line resources by the same author [1], we were able to create a fully parametric model of a Moineau pump (rotor and stator) with an (almost) arbitrary number of lobes $n$ using the OpenSCAD 3D modelling language [4]. The OpenSCAD files have been submitted to the online repository of 3D models "Thingiverse" [5] under the Creative Commons - Attribution license.

After this first (mostly theoretical) analysis, we started to search for an optimal way to implement the Moineau pump to build a volumetric plastic extruder to be used in a RepRap FDM 3D printer. Usually, the implementation of an standard Moineau pump involve the rotor made of a solid surface (steel coated with chromium), with the stator made of elastomer inside a metal tube of complex shape to form the inner cavities that roll around. Our device model is shown in Fig.1. 


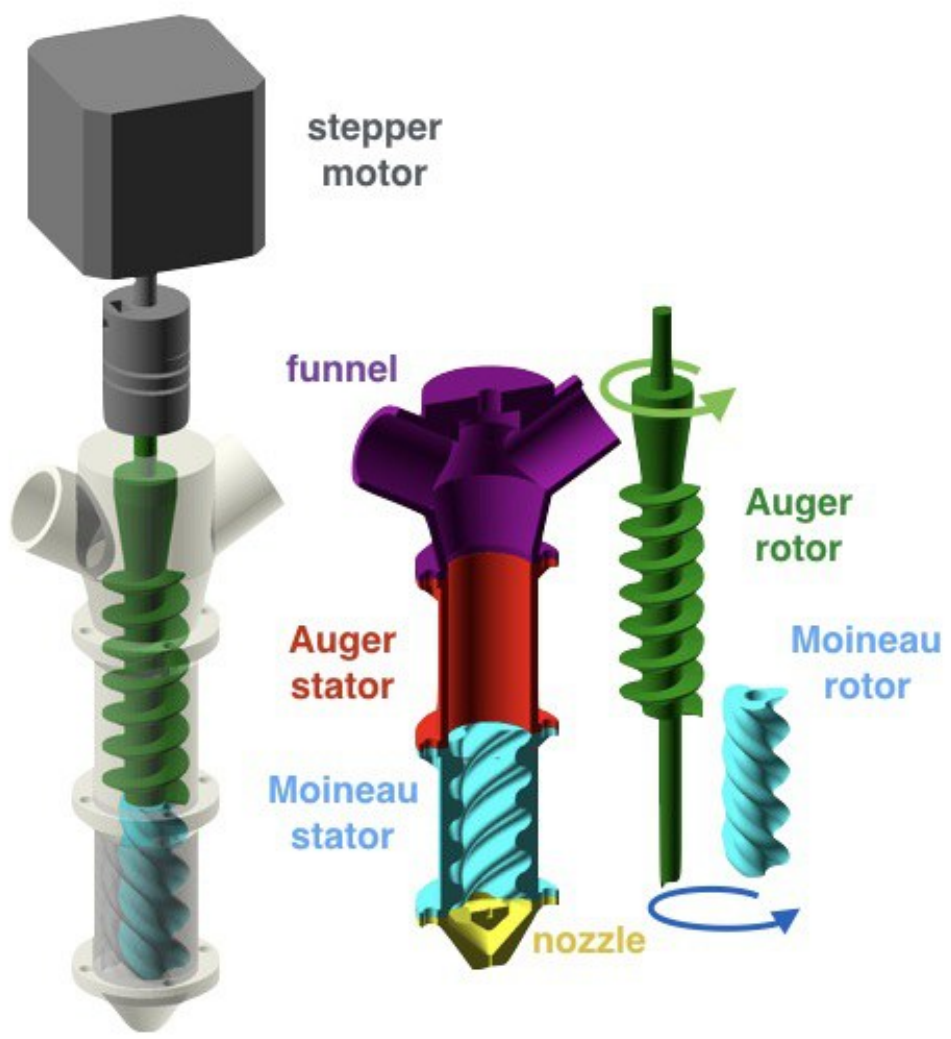

Figure 1: Schematics of our pellet extruder, combining together Auger and Moineau screws.

In our case, the Moineau pump alone needs to be fed by a conveyor with the ability to transport industrial-type plastic pellets (and/or granules) and melt them. We decided therefore to combine it with an Auger pump (Archimede's screw) as first stage. The main problem with such combination is that the Auger screw has an axially-centered rotation, while the Moineau rotor movement inside its stator is an eccentric rotation (off-axis). This problem has been solved by using a crankshaft-type coupling: the Moineau rotor is driven by an axis prolonging off-center from the Auger screw, that is itself driven by a motor. A critical detail is the second centering point located after the Moineau rotor, where the "nozzle" stage is attached (see Fig.1). All files for such mechanism have been also submitted to the online repository of 3D models "Thingiverse" [6] under the Creative Commons - Attribution license.

Following this initial design we iterated a few more times while solving other technical issues, so that the first working prototype is composed by multiple independent stages (modular design) and has been 3D printed out of Aluminum by using an on-line commercial 3D-printing service (as shown in Fig. 2(a)). This very first prototype, after some initial test with a manual crank, was modified to work with a stepper motor coupled with the extruder axis. The stator section was connected to the stepper motor unit using some mechanical flanges milled with a CNC machine.

During the assembly we noticed that it was difficult to properly align the Auger-Moineau axes: the rotation was badly affected by very little shifts of the axes from the exact position and the coupling with the stepper motor was not solid enough to guarantee the correct alignment. Another critical element was the absence of a fixed centre of rotation on the bottom side of the Moineau rotor, where the "nozzle" stage is attached: in the previous plastic prototype the Moineau rotor was often blocked due to loss of parallelism between the rotor and the stator axes because of excessive gap; this issue was subsequently avoided by adding a small shaft protruding from the eccentric Moineau rotor axis, and rotating inside a hole in the centre of the nozzle section. Even if the first aluminum 3D-printed prototype had a remarkable dimensional precision, the little anchor on the nozzle-side of the axis was impossible to print with Direct Metal Laser Sintering technology because of its tiny diameter. To solve this problem we drilled the axis to create a pocket in which we glued a tiny steel rod with a diameter of one millimetre; this solution was acceptable at low temperature but has not survived to the following tests at higher temperature (200 degrees Celsius and above). During these preliminary tests with PET pellet using the first aluminum prototype, we noticed that the plastic was melted only in the lower part of the Auger screw (see upper Fig.2(a)), because only one heater was positioned on the Moineau section. The add of a second heater in the Auger section can provide a better control of the temperature on the extruder. This first prototype has been 
tested at low temperature (below 60 degrees Celsius) with good success, using pellets of wax and chocolate as displayed in Fig.2(b), but we have not been able to test it yet with plastic pellets for the reasons explained above.
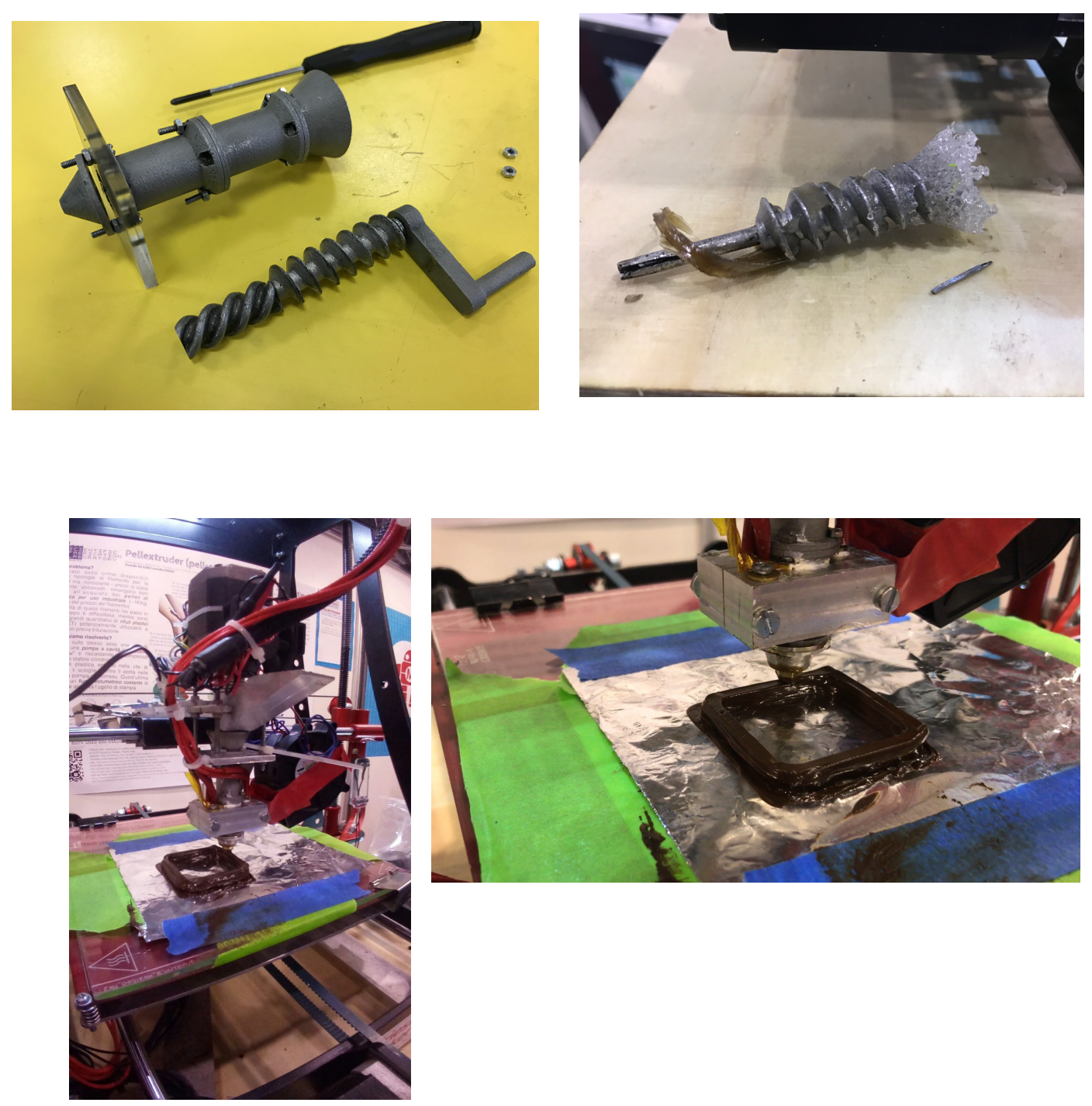

Figure 2: (a) Upper pictures: (left) first Pellextruder, 3D printed in Aluminum with manual crank; (right) plastic pellets melted only in the lower part of the Auger screw due to the high temperature gradient along the path.

(b) Successufuly working extrusion of chocolate and wax pellets below $60^{\circ}$ Celsius are shown in the images below.

A second working prototype was then developed: the entire axis was redesigned to permit the production directly from a unique piece of steel with a milling machine, and some of the dimensions were also modified. In this way the little centering rod is more resistant and the Auger screw can be produced independently from the axis. The coupling with the stepper motor remains a part of the Auger rotor piece, but to reduce the friction and simplify the axis positioning a bearing has been added on the feeder-side of the extruder. In order to maximise the diameter of the centering rod between the Moineau and the nozzle, it was necessary to increase the number of lobes of the Moineau rotor, from $n=3$ to 4 .

This second model aims to solve also another problem that afflicted the first prototype: a single NEMA-17 stepper motor could not provide enough torque to extrude dense pellets. In order to limit the extruder dimensions and weight we decided not to use a larger stepper motor but to increase the torque using a geared reduction mechanism. With a more resistant steel axis and a higher torque, this new prototype (yet under construction) should work also with plastic pellets, that are harder than the chocolate and wax granules used in our first tests with the first prototype of Fig.2. Finally, we observed that a large quantity of pellets could block the Auger screw in the upper part. This is not a common situation, but it can be easily avoided extending the screw length up to the upper bearing, which is the maximum distance that can be used. 
Based on these crucial experiences, we also worked out a way to look at the whole extruder device from a new perspective. For this purpose we have developed alternative Moineau-based pumps for the volumetric extrusion of pellets as presented next.

\section{Alternative Layered Moined-based pump}

In order to design an alternative multi-layer pump system that could be easier to build, assemble, and clean, and that could get in practice a reasonable (approximated) continuous pumping of viscous fluid, we started analysing in deep a classical (2:1) Moineau as depicted in Figure 3.

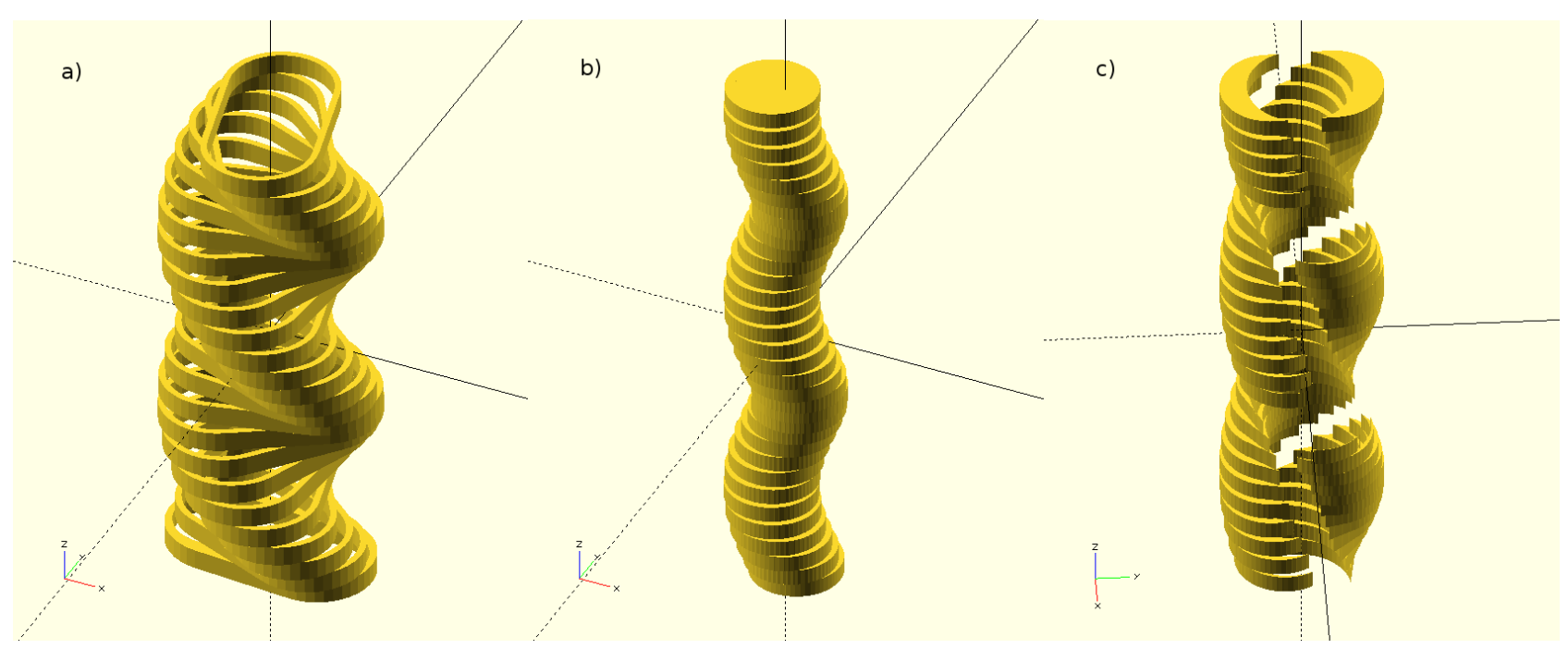

d)

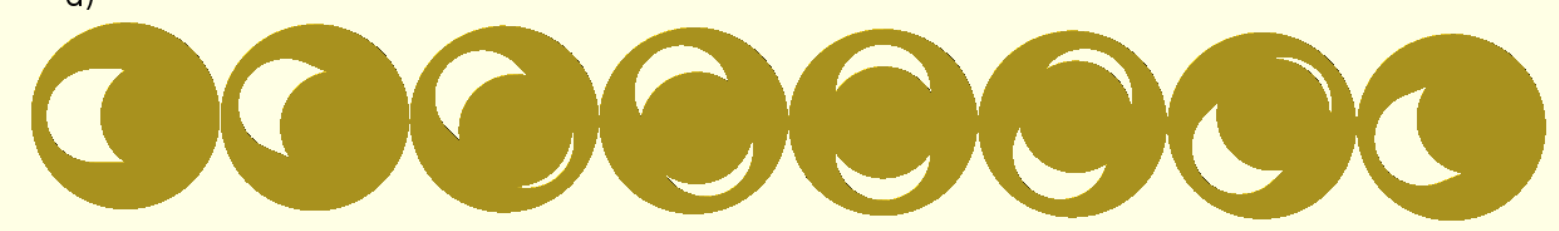

e)

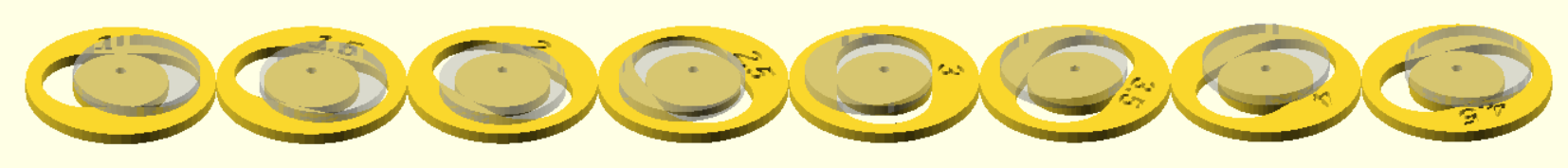

Figure 3. Moineau pump with a twist of $450^{\circ}$ subdivided in layers: (a) stator, (b) rotor, (c) inner gap (cavity), (d) inner mechanism along $180^{\circ}$ at $45^{\circ} / 2$ intervals, and (e) circular motion around the stator center and layers' labelling every $90 / 5$ angles in our model.

In Figure 3(a) and (b), a progressive cavity pump, having a single-helix hard rotor and a double-helix stator (of 5/6+5/6 the width of the rotor diameter), are shown subdivided in several, equally spaced, horizontal layers for one twist of 450 degrees. Figure 3(c) illustrates the material flow in the cavities (or gap) throughout the Moineau. Figure 3(d) illustrates from the top, the Moineau inner mechanism through one full revolution of $180^{\circ}$ (i.e., one pitch) at $45^{\circ} / 2$ intervals, which repeats itself up to a total angle of $450^{\circ}$. From this figure it can be seen immediately that the (circular section of the) rotor at any chosen layer translates linearly back and forth across the stator opening as it is rotated within the fixed stator. This occurs because of a combination of the rotation of the rotor around its own centroidal axis in the clockwise direction and eccentric reverse rotation (i.e., nutation) of the rotor about the centroidal axis of the stator [7]. In figure 3(e) we labelled the layers by starting with number "1" for $0^{\circ}$ rotation, "1.5" for $45^{\circ} / 2$, "2" for $45^{\circ}$, "2.5" for 45 $\mathrm{x}(3 / 2)$, "3" for $90^{\circ}$ rotation and so on. The center of the stator is also shown together with the movement of a given (secondary) circle in transparent grey, that can rotate around the stator centerline in every layer. It is important to observe that each 
grey circle rotates (in a perfectly circular motion) around the centraline of the stator provided its axis is displaced a dis tance of $1 / 3$ the radius of the rotor. By rotating this grey circle with such off-axis, the stator can also get open in two di rections in a rather similar way as the rotor does in Fig.3(d) (for a formal mathematical description and more details of this dynamics see [7]). We use this last feature to build upon a simplified layered Moineau based pump.

After the analysis of these longitudinal cuts, we re-designed an alternative multi-layer pump system simplified to just six layers that are easier to build and assemble in order to get in practice, as shown in the next section, a reasonable continuous pumping of viscous material. The reduced system consists of selecting six layers of the stator as illustrated in Fig.4(a) in color blue.

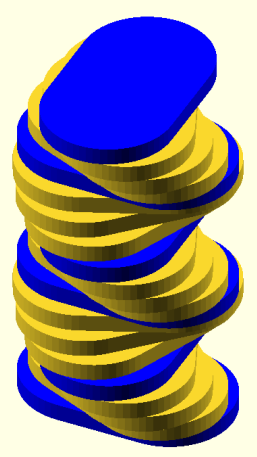

a)

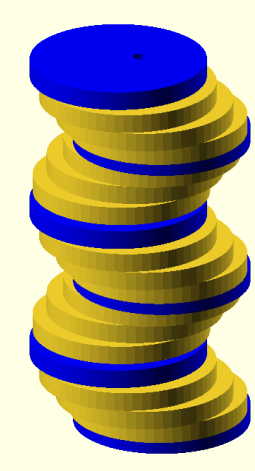

b)

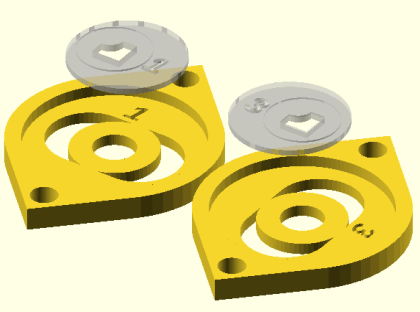

c)

Figure 4. Pump model 131313: (a) stator labels "1" and " 3 " in blue twisted by $90^{\circ}$ up to $450^{\circ}$ same as the Moineau pump, (b) "equivalent" rotor layers, formed by means of the grey circles of Fig.3(e), (c) selected "1" and "3" layers having the forms ready for 3D printing (in .STL format).

In Figure 4(a), the inner (empty) part of our stator layers is only shown in yellow to facilitate the viewing of this selec tion. We have chosen the stator cross-sections "1" and "3", because when we put together one on top of the other 3 times each, they twist for a total angle of $450^{\circ}$ same as in the layered Moineau pump of Fig.3(a). The corresponding six layers of the "equivalent" rotor, formed by means of the grey circles of Fig.3(e), are shown in Fig 4(b). Thus, we have then designed a simple screw-like model out of these 131313 layers selected having the forms of Fig.4(c). Hence the name for this model.

\section{Discussion}

Our prototypes for Moineau-based pumps, shown in Figs. 1 and 4, have the main final goals of $i$ ) extruding plastic pellets at high temperatures, and ii) having a volumetric control of the extrusion flow at these high temperatures.

Our research follows previous works, which developed plastic pellet-fed extruders based on Auger screws only. These have been able to extrude successfully and 3D-print whole objects, but the absence of a precise volumetric control of the extrusion is a limiting factor for the type of objects that are printable: only "spiralize" technique is possible, without the use of filament retraction, therefore allowing the creation of simple models and only after some manual tuning of the auger rotation speed, according to the current temperature and viscosity of the melted plastic. An example of such technology is the DeltaWASP 3MT printer [9]. For this reason we decided to explore the use of Moineau pump technology to add precise volumetric control to the extrusion of pellets. It is important to mention that a Moineau pump has to be coupled to a first stage Auger screw, in order to fed with melted plastic without inclusion of air bubbles, since the Moineau pump itself cannot guarantee such condition.

There are several types of Moineau pumps, according to their geometry [1]. In particular, the number of lobes plays a critical role in defining the overall characteristics of the pump. To decide which would be the most appropriate number of lobes for the prototype to be used in our tests of the Moineau pump, we carried out a quantitative analysis on the vo lumes and surfaces of multi-lobe Moineau pumps. As shown in Fig. 5, we have found that there is a logarithmic dependency of such quantities with the number of lobes. To achieve these findings, 3D models of the Moineau rotor and stator have been developed using OpenSCAD parametric 3D-modelling software [9], and the volume and surface (in arbitrary units) of the progressing cavities has been estimated for different values of the number of lobes using the MeshLab open source software.

From this analysis, we decided to use a small number of lobes $n$ for the Moineau rotor ( 3 and 4 ) because of the constraint given by the minimum possible diameter for the axes. Nicely we have found that this choice maximises the volume of the cavities and therefore the amount of extruded material per single rotation. The fact that this choice also mini - 
mises the surface of such cavities implies that the tolerance problems between rotor and stator are minimised, even without the use of elastomer parts as gaskets (we use only aluminum parts due to our high temperature requirements for the extrusion of plastic pellets).

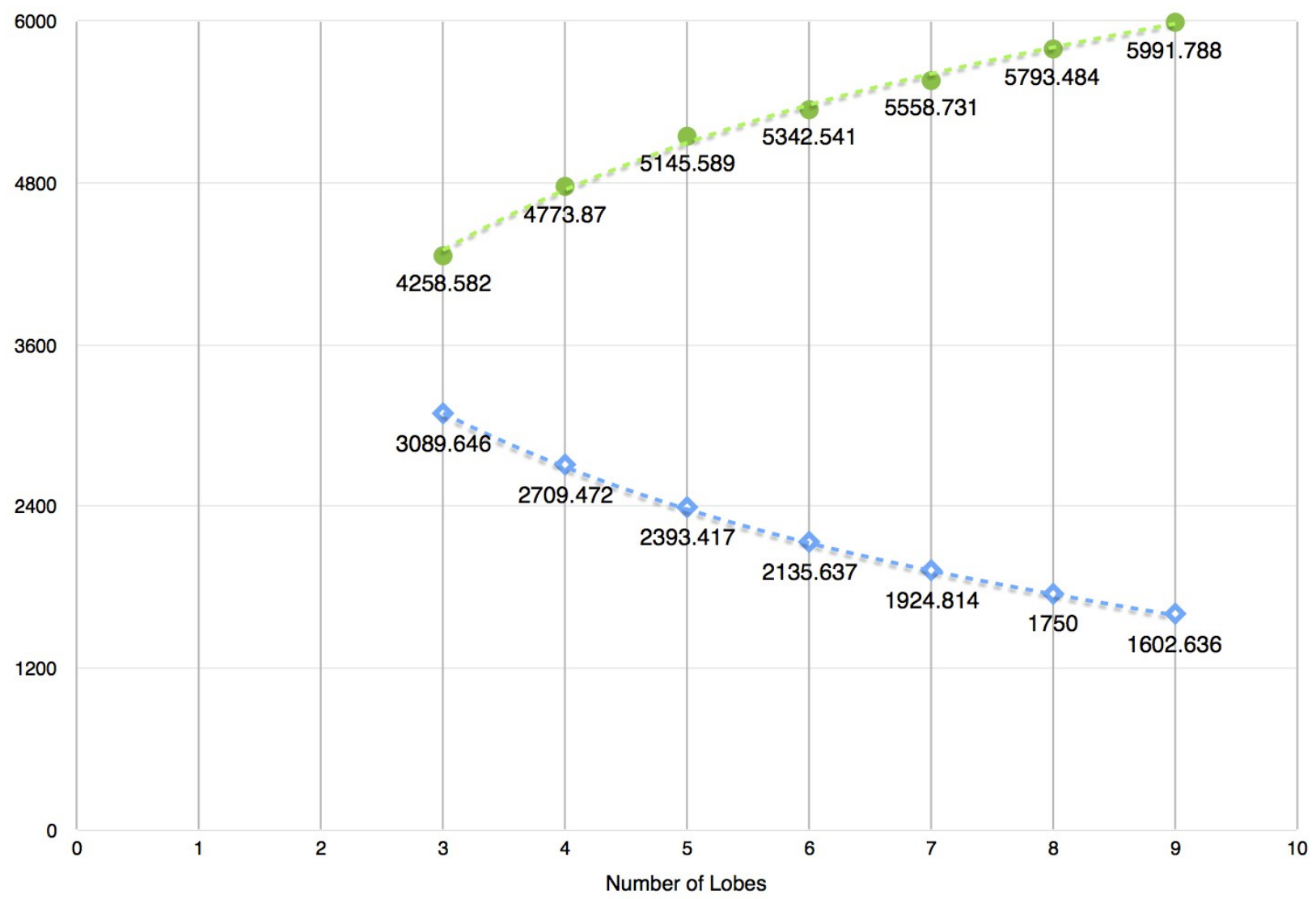

Figure 5: Volumes and surfaces of multi-lobe Moineau pumps (in a.u.).

To take into account the need for a gap between the moving parts, we had to model the rotor and stator shapes in a slightly different way respect to ordinary Moineau geometry. This has been solved by scaling-down by $0.1 \mathrm{~mm}$ the radii of the epi- and hypo-cycloids that generates the stator (therefore subtracting a small offset all around the shape), and at the same time by reducing the rotor shape in the tangential direction without affecting the radial component. To do the latter we computed the intersection of the rotor with a rotated copy of itself (amount of rotation: 1 degree). In Fig. 6 . the small corrected rotor-stator gap of the (5:4) Moineau pump is shown. We worked out this empirical solution after having issues with the tolerances from the first 3D-printed prototype in aluminium.

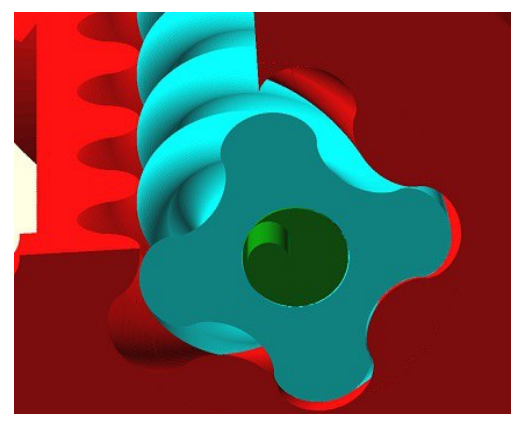

Figure 6: View of the tiny gap between the rotor (in blue) and the stator (in red) of the $(5: 4)$ Moineau pump 
On the other hand, we tested the 3D printed prototype in PLA for the simplified pump model 131313 og Fig. 4 based on the Moineau mechanism. In Figure 7 we show the 3D printing parts of the six layers that form the screw-like pumps of Fig.8. The dimensions of each layer types "1" and "3" are $2 \mathrm{~mm}$ in height and $30 \mathrm{~mm}$ in diameter, surrounded by a tiny border of another $2 \mathrm{~mm}$ in height to contain the corresponding layered stator of heigh (25\% less than) $2 \mathrm{~mm}$ (smaller to reduce friction during its rotation). The steer Auger portion has a conical shape and it is placed at the top to increase pressure as the layered rotor-like is turned. As mentioned, it is used as conveyor to fed viscous material into the system.

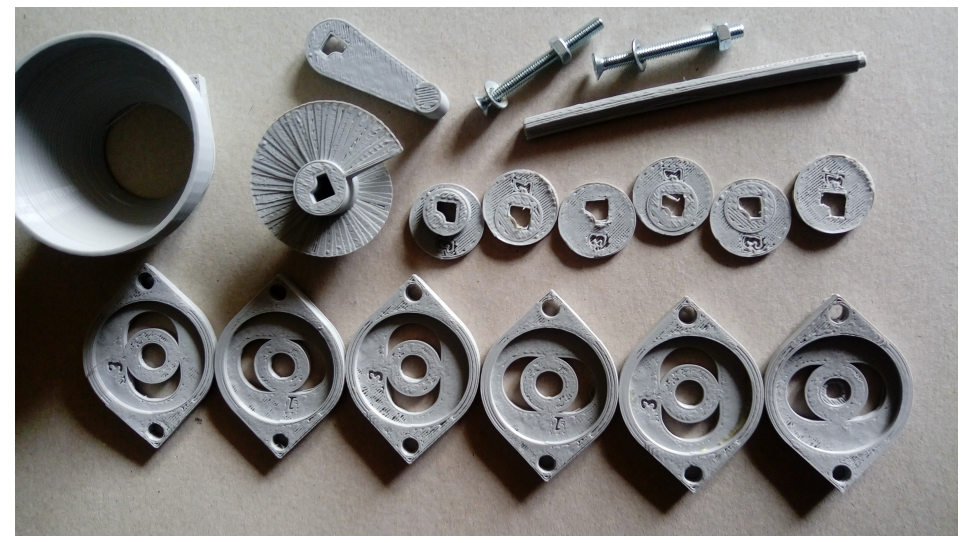

Figure 7. 3D printing parts of the six layers that form our screw-like pump
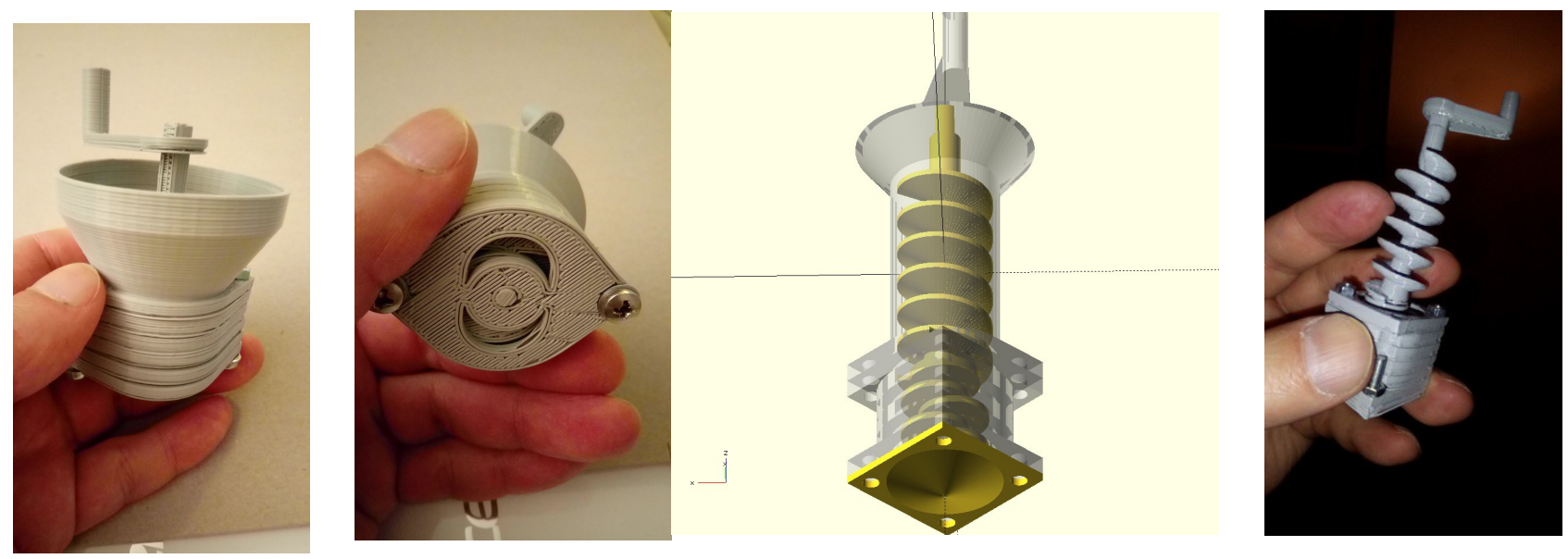

Figure 8. Different side views of our pump model 131313 initially 3D printed in PLA for testing purposes. On the left, the one having a short conveyor auger screw on the top, and on the right a twice larger auger screw to increase pressure as the layered rotor-like is turned manually by a crank or a stepper motor.

The first 3D printed prototype of the screw-like pump model 131313 of Fig. 8 allowed us to test the extruder using a viscous material at room temperature. As the layered rotor is turned by the external crank, this simplified pump can displace a fixed quantity of fluid contained in the layered stator in vertical direction by means of the co-axial Auger screw on top, with a quantity of material that depends linearly on the velocity of rotation. This extraordinary behaviour can be seen in action by watching our YouTube videos in [9] entitled "Layered screw-like pump: Model 131313 by@SciFabLab" and "Mini Extruder: Layered Moineau based Pump plus Auger". The two representative video frames in Fig.9 illustrates the alternating switch of the extrusion between the two sides by turning the layered rotor, as well as the performance of the expelled fluid by our pump model 131313.

In Figure 10 we plot the quantity of units of expelled material (i.e., approx. volumetric flow rate $Q$ ) versus the number of turns of the rotor for a full charge of material contained in the pump (i.e., approx rpm $N$ ). Having the dimensions described above, the pump gets completely empty after approximately 120 spins of the crank. The curves in the figure are the linear fitting to the left-side (red dots) and right-side (blue dots) extrusions. This behaviour shows that in our model 131313 the units of extruded material becomes reasonably proportional to the number of spins of our layered rotor (i.e., approx. rotation rate). The material flow can only be controlled unidirectionally $\neg$ which is an enough condition for some applications like vertical 3D printing. 

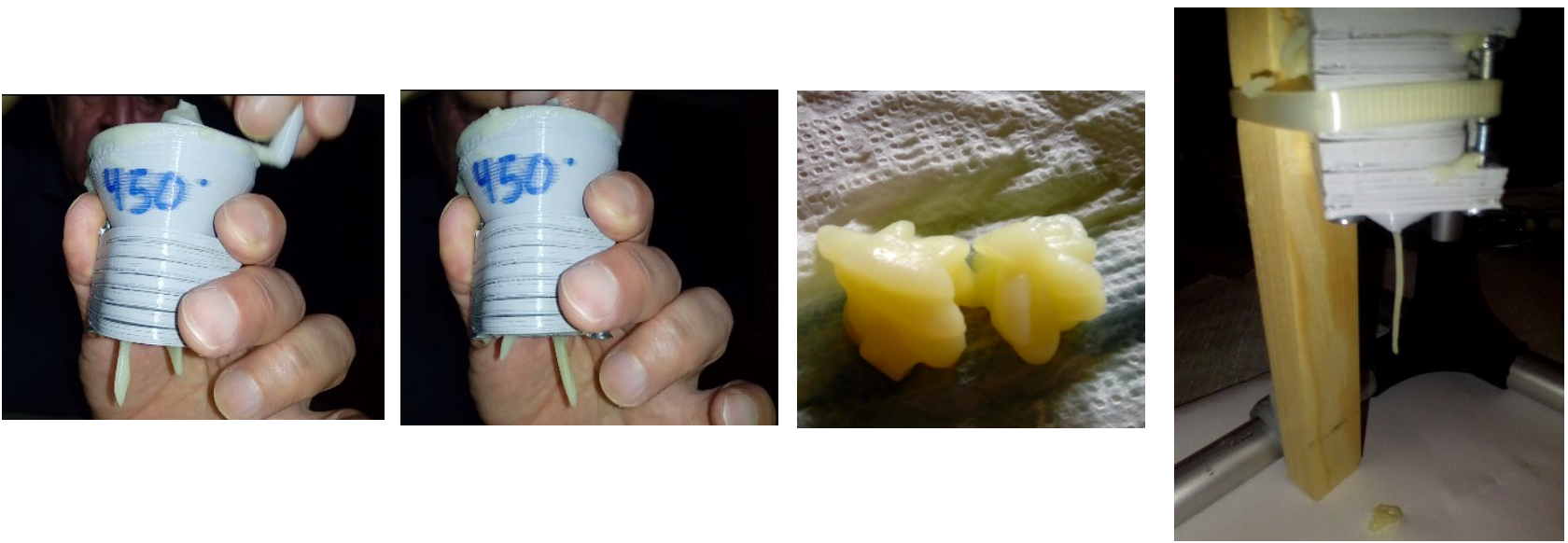

Figure 9. Alternating extrusion and performance of the pump model 131313 (snapshots from YouTube videos in [9]).

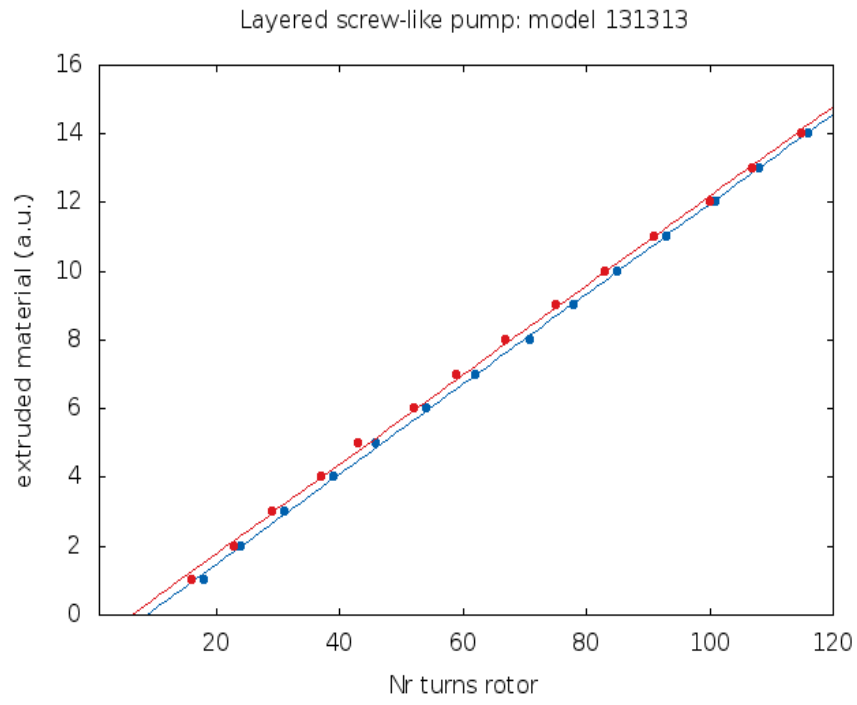

Figure 10. Quantity of units of expelled material vs. the number of turns of the layered rotor for a full charge of material.

To conclude we mention that in all the models of Moineau-based pumps studied in this work consist of an stator and rotor coupled with an Auger screw. These parts can be scaled to small dimensions and can be 3D printed in metal. As a main difference between our first working Pellextruder, 3D printed in Aluminum of Fig. 2, the alternative layered model 131313 of Figs. 4 and 7, is that in the latter the Auger screw is co-axial, it has a conical form, and it is placed inside the top container of the viscous material (to consist of pellets at high temperatures), making it half shorter. This device, made of an discretised stator and equivalent discretised rotor, is thus compact and it consists of few layers only.

\section{Concluding Remarks}

In an attempt to develop a low-cost solution for an environmental-friendly and open source 3D printer, the (heated) volumetric extrusion mechanism driven by our Moineau-based screw pumps could found application in the production of 3D objects directly from thermoplastic pellets or granules of recycled plastic rather than from filament by adapting the nozzle mechanism of 3D RepRap printers. In fact, the higher cost and lower availability of these plastic filaments make FDM 3D printers under-utilised in developing countries, where they may be of paramount importance to foster local development and sustainability (i.e., by 3D printing of mechanical spare parts, rapid prototyping of new products, educational models, research and Lab equipment, additive manufacturing, etc).

The first prototype of the Moineau pump of Fig.2, fabricated in aluminium, allowed us to test the extruder using semiliquid paste as was done previously with the same model 3D printed in PLA at room temperature. The initial crank mechanism has been replaced with a stepper motor and an additional support structure has been added to keep all parts together. With a second aluminum prototype we are solving several problems that afflicted the first prototype, with improvements like a redesigned steel axis coupled with the stepper motor through gears and bearings, a longer Auger screw and a more precise control of temperature on the entire length of the extruder. The results found so far (chocolate, 
wax) with the first aluminum prototype of our extruder are encouraging, and many other tests are planned with the second prototype that is now under construction (3D-printing parts in aluminum takes long time to get, cosidering the complexity of our device).

On the other hand, our alternative simplified 3D printed prototype of the screw-like pump model 131313 of Fig.4 allowed us to extrude a viscous material at room temperature so far. In fact, it can displace constant material depending of the velocity of rotation (see YouTube videos in [8]).

A main challenge is the need for keeping these systems at high temperatures (say, 180-to-250 Celsius degrees) to handle melted plastic instead of commercial filaments. Such extrusion system would allow also to reu se the plastic material from 3D-printed support structures or from any other discarded 3D-printed model for the benefit of a clean society. Once these pumps will become fully operative, a real estimation of their performances in terms of relative flow rate Q, speed (rpm) $\mathrm{N}$, head $\mathrm{H}$ and power $\mathrm{P}$, will follow by the relations $Q_{1} / Q_{2}=N_{1} / N_{2}, H_{1} / H_{2}=\left(N_{1} / N_{2}\right)^{2}, P_{1} / P_{2}=\left(N_{1} / N_{2}\right)^{3}[10]$.

By modifying the nozzle mechanism of 3D RepRap printers, the rotary positive displacement pumps studied 7 where the fluid is displaced axially at a constant rate, could allow to reduce the $3 \mathrm{D}$ printing costs by $1 / 3$ and to enlarge the adoption of 3D printing technologies by millions throughout the developing world. Work along these lines is still in progress in our SciFabLab [11], with the final goal to share this knowledge and collaborate across international borders in the fields of science, education and sustainable growth using open tecnologies as the one at hand.

\section{Acknowledgements}

Our sincere thanks go to all members of the ICTP SciFabLab for their many suggestions and help during the development of this project.

\section{References}

[1] J. Gravensen, "The geometry of the Moineau pump", Computer Aided Geometric Design, 25 (2008) $792-800$. http://www2.mat.dtu.dk/people/J.Gravesen/pub/30-2008.pdf Additional on-line resources by the same author at website: https://web.archive.org/web/20150912184453/http://siags.siam.org/siaggd/problems/gravesen/

[2] US patent 8597386 B2: https://www.google.com/patents/US8597386

[3] RepRap Magazine - website: www.reprapmagazine.com

[4] OpenSCAD free software for creating solid 3D CAD objects - website: www.openscad.org

[5] Thingiverse on-line repository of 3D models - website for our theoretical study of Moineau pumps: www.thingiverse.com/thing:1665474

[6] Thingiverse on-line repository of 3D models - website for our Moineau + Auger pumps: www.thingiverse.com/thing:1689212

[7] Progressing cavity pumps: Fundamentals -website: www.pc-pump.com/pcp-systems/fundamentals see also: www.petrowiki.org/Downhole_PC_pumps

[8] DeltaWASP 3MT printer at www.wasproject.it/w/en/wasp-presents-deltawasp-3mt-in-england-and-united-states

[9] YouTube videos: "Layered screw-like pump: Model 131313 by@SciFabLab", www.youtube.com/watch?v=qcMlMf31JU0 and "Mini Extruder: Layered Moineau based Pump plus Auger" www.youtube.com/watch?v=Y3TjkRAStZk

[10] Iowa State University's Center for Industrial Research and Service:"Energy-Related Best Practices: A Sourcebook for the Chemical Industry" (2005): www.ciras.iastate.edu/publications/EnergyBP-ChemicalIndustry/

[11] C. Fonda and E. Canessa, "Making ideas at scientific fabrication laboratories", Phys. Education, 51 (2016) 065016 